\title{
Formulation and Evaluation of Gastro-bilayer Floating Tablets of Ezetimibe as Immediate Release Layer and Atenolol as Sustained Release Layer
}

\author{
Ranjit Prasad Swain, Avasarala Nagamani, Panchagnula Udaya Shankar \\ Department of Pharmaceutical Technology, Maharajah's College of Pharmacy, Vizianagaram, Andhra Pradesh, INDIA.
}

\begin{abstract}
Introduction: Combination therapy of ezetimibe and atenolol is highly desirable for better management of dyslipidaemia and hypertension. Ezetimibe has poor solubility hence variable and low bioavailability. Atenolol has poor absorption in lower gastrointestinal tract, short half-life. Therefore, the present study was to develop gastro-bilayer floating matrix tablet in which ezetimibe was incorporated as immediate layer and atenolol as sustained release layer. Methods: Solubility of the ezetimibe was enhanced by solid dispersion technique and was characterized by FTIR, DSC and XRD study. Gastrobilayer floating tablets were prepared by direct compression method. Results and Discussion: Hydroxypropyl methylcellulose $\mathrm{K} 100(37.5 \% \mathrm{w} / \mathrm{w})$ as release retardant and croscarmellose sodium $(15 \% \mathrm{w} / \mathrm{w})$ as superdisintegrants in immediate layer as optimized. The total floating time of the optimized tablet was $12 \mathrm{~h}$ with 9 min of floating lag time. Atenolol release was sustained through diffusion mechanism over $12 \mathrm{~h}$ and more than $95 \%$ ezetimibe was released within $30 \mathrm{~min}$. Conclusion: It can be concluded that biphasic drug release pattern was successfully achieved through the formulation of gastro-floating bilayer tablets in this study, allowing strengthened combination therapy for hypertension and dyslipidemia.
\end{abstract}

Key words: Gastro-bilayer, floating, immediate release, sustained release, ezetimibe, atenolol.

\section{INTRODUCTION}

Frequent dosing in oral drug delivery results in fluctuation of plasma drug concentration and finally toxicity. ${ }^{1}$ To overcome this problem oral controlled drug delivery system was developed that deliver the drug for an extended period of time. Physiological problems like drugs with narrow absorption window, alteration in emptying time of stomach, drugs that has stability issues in intestine and drugs that are transported via active transport mechanism ${ }^{2}$ were the difficulties of this system. To overcome these difficulties gastroretentive drug delivery system (GDDS) has been developed. Gastroretentive systems can remain in the gastric region for several hours and prolong the gastric residence time of drugs. Prolonged gastric retention improves bioavailability, reduces drug waste and improves solubility for drugs that are less soluble in a high $\mathrm{pH}$ environment. It also provides local drug delivery to the stomach and proximal small intestine. ${ }^{3}$ Among the gastroretentive dosage forms, floating drug delivery system is considered to be most favourable because it does not intensely affect the motility of GIT. ${ }^{4}$ The matrix bilayer tablet with two separate release-layers is a biphasic delivery system that aims to deliver drug at two different rates or simultaneously releases two drugs with the benefits of formulating two chemically incompatible drugs into a system, simultaneously releasing two APIs with desired release profiles, increasing efficacy of API by a synergistic effect,
Submission Date: 12-10-2018; Revision Date: 23-11-2018; Accepted Date: 29-12-2018

DOI: 10.5530/ijper.53.2s.53 Correspondence: Dr. Ranjit Prasad Swain, Department of Pharmaceutical Technology, Maharajah's College of Pharmacy, Vizianagaram-535 002, Andhra Pradesh, INDIA. Phone: +919701543797 E-mail: ranjit.prasad797@ gmail.com

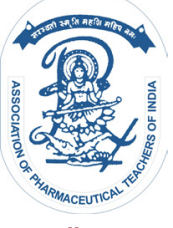

www.ijper.org 
decreasing the dosing unit burden and better patient compliance. ${ }^{5}$

Cardiovascular disease is a collective term that covers any disease of heart and circulatory system. ${ }^{6}$ The foremost risk factors for cardiovascular diseases are tobacco exposure, hypertension, high cholesterol, alcohol consumption, obesity. Globally cardiovascular diseases accounts for approximately 17 million deaths a year of these 9.4 million deaths are due to hypertension. ${ }^{7}$ The first National Health and Nutritional Examination Survey (NHANES) estimated the co-prevalence of hypertension and elevated total cholesterol. During the third NHANES, around $60 \%$ of adults had concomitant hypercholesterolemia and $55 \%$ of the adults with hypercholesterolemia had hypertension. ${ }^{8,9}$ Combination therapies that target several risk factors, such as hypercholesterolemia, hypertension, diabetes and artery function may more effectively treat cardiovascular disease than a therapy that targets several risk factors for the cardiovascular death. ${ }^{10-12}$ So, combination therapy is required to deliver drugs for dyslipidaemia and hypertension.

Atenolol is a cardio-selective $\beta-1$ adrenoceptor devoid of intrinsic sympathomimetic and membrane stabilizing activity. It belongs to anti-hypertensive category and therefore reduces the high blood pressure. ${ }^{13}$ It has absorption window in upper GIT and the poor absorption in lower GIT. This varied absorption results in lowering bioavailability i.e., $50 \%$ with a half-life of 6-7 h. ${ }^{14}$ Thus, it seems that increase in gastric residence time may increase the extent of absorption and bioavailability of drug. Hence, it was selected as sustained release layer to improve bioavailability. Ezetimibe is classified under anti-hyperlipidemic class and which is a BCS class II drug. ${ }^{15}$ So, the solubility must be improved. It has longer biological half-life $(22 \mathrm{~h})$ and its bioavailability was 30-60\%. ${ }^{16}$ So, it can be formulated into an immediate release layer to achieve better absorption. The poor level of control of hypertension and dyslipidemia highlights the need for new strategies to manage these risk factors thereby reducing the impact of cardiovascular diseases. A single pill combination of an antihypertensive and lipid lowering medication may address some of the issues through to hinder the management of cardiovascular diseases, such a poor adherence to multiple treatments due to high pill burden and the reluctance of physicians to manage more than one of cardiovascular risk factors simultaneously. Swain et al. ${ }^{17}$ and Kulkarni et al. ${ }^{18}$ worked on development and evaluation of bilayer floating tablets of atenolol and simvastatin/lovastatin for biphasic release profile to improve the bioavailability of atenolol and to treat hyperlipidemia which is the major problem in hypertensive patients. ${ }^{4}$ Ezetimibe is a lipid - lowering agent acts immediately and reduces the cholesterol absorption and then atenolol, an antihypertensive agent sustains its action over longer period of time which may useful for management of cardiovascular diseases. To the best of our knowledge, no reported literature was found on the combination therapy of ezetimibe and atenolol. In the present study we have made an effort to formulate a gastro-bilayer floating system of ezetimibe in the immediate release layer and atenolol in the sustained release layer to improve their bioavailability.

\section{MATERIALS AND METHODS}

\section{Materials}

Atenolol was procured from Yarrow chem products (Mumbai, India). Ezetimibe was a gift sample from Aurobindo Pharma Pvt. Ltd., India. Hydroxy propyl methyl cellulose, PVP K- 30, PEGs, croscarmellose sodium were supplied by Yarrow chem. Pvt. Ltd., Mumbai. Sodium bicarbonate and lactose were purchased from Thermo Fischer Scientific Pvt. Ltd. (Ahmedabad, India). Microcrystalline cellulose was obtained from Himedia (Gurgaon, India). Ethanol and $\mathrm{HCl}$ were from Fischer Scientifica, India and of analytical grade.

\section{Methods}

\section{Solubility enhancement of Ezetimibe}

Solubility of ezetimibe was enhanced by preparing solid dispersions (SDs) by solvent evaporation method (SM) using PVP K-30 (ESD1), PEG 6000 (ESD2) and PEG 8000 (ESD3) in the ratio of 1:1 w/w (drug: carrier). Ethanol $(10 \mathrm{~mL})$ used as solvent to solubilize the ezetimibe and polymers by continuous stirring with a magnetic stirrer (RemiElektrotechnik Limited, India, Model: $1 \mathrm{MLH})$ for an hour at room temperature. The mixture was stored at a room temperature for complete evaporation of ethanol. The resulting SDs were scraped, pulverised and passed through $40 \#$ mesh sieve. The samples were stored in screw cap glass vials and kept in desiccators until further analysis..$^{19,20}$

\section{Characterization of SDs}

\section{FTIR spectroscopy}

FTIR spectra of ezetimibe, PVP K-30 and SD were subjected to compatibility studies by diamond ATR spectrophotometer (Cary 60, Agilent technologies, Germany). The prepared SDs were scanned at a resolution of $2 \mathrm{~cm}^{-1}$, from 4000 to $400 \mathrm{~cm}^{-1}$. 


\section{Differential scanning calorimetry (DSC)}

Thermal analysis was carried out for ezetimibe, PVP K-30 and SD using DSC (Pyris Diamond, Singapore). The samples were heated at a constant rate of $10^{\circ} \mathrm{C} / \mathrm{min}$ over a temperature range of $30-250^{\circ} \mathrm{C}$ using platinum crucible with alpha alumina powder as reference to predict any physicochemical interactions between components.

\section{X-ray diffraction analysis (XRD)}

Ezetimibe, PVP K-30 and SD were subjected to X-ray diffraction analysis, using $\mathrm{Cu}$ target slit $10 \mathrm{~mm}$ (ULTIMA III, Japan) to investigate physical state of SD.

\section{In vitro dissolution studies}

Dissolution studies were performed for the SD using USP dissolution type II apparatus (paddle method) with $50 \mathrm{rpm}$ in $900 \mathrm{ml}$ of $0.1 \mathrm{M} \mathrm{HCl}$ as dissolution medium at $37 \pm 0.5^{\circ} \mathrm{C}$. The samples were withdrawn at predetermined time intervals $(5,10,15,30,45$ and $60 \mathrm{~min})$ and the same amount of preheated $\left(37 \pm 0.5^{\circ} \mathrm{C}\right)$ fresh medium was added to maintain constant volume throughout study. The percentage drug release values obtained from the dissolution studies were plotted against time.

\section{Compression of immediate release tablets}

SDs equivalent to $10 \mathrm{mg}$ of ezetimibe was blended with croscarmelose sodium and lactose to prepare IR1, IR2, IR3 formulations and passed through sieve 44\# then added magnesium stearate before direct compression of tablets (Table 1).

\section{Compression of sustained release tablets}

All the ingredients of formulations (SR1-SR9) shown in Table 2 were accurately weighed and passed through sieve 44\# to obtain uniform size. Magnesium stearate
Table 1: Formulation of immediate release layer tablets by direct compression method

\begin{tabular}{|c|c|c|c|c|c|}
\hline 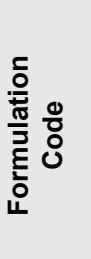 & 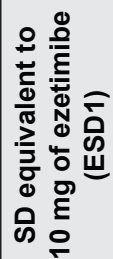 & 乌ુ & 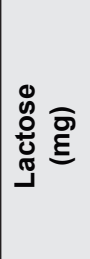 & 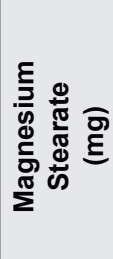 & 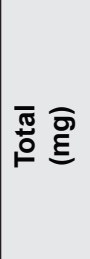 \\
\hline IR1 & 40 & 5 & 54 & 1 & 100 \\
\hline IR2 & 40 & 10 & 49 & 1 & 100 \\
\hline IR3 & 40 & 15 & 44 & 1 & 100 \\
\hline
\end{tabular}

was added before punching of tablets by direct compression method.

\section{Preparation of gastro-bilayer floating tablets}

Immediate release tablets (IR3) and sustained release tablets (SR9) were selected based on dissolution profile. The gastro-bilayer floating tablets were prepared with varying concentration of sodium bicarbonate $(3.5,5.0$, $6.25,7.5 \% \mathrm{w} / \mathrm{w}$ ) in the sustained release layer (Table 3). Bilayer floating tablets were formulated with multistation rotary tablet punching machine via single compaction method. Initially, sustained release layer powder blend was accurately weighed and fed into die cavity of tablet punching machine. Similarly, immediate release layer powder was fed to die cavity which was previously filled with the sustained release layer and compressed so that the final hardness obtained for the bilayer floating tablet was between $5.5-6.5 \mathrm{~kg} / \mathrm{cm}^{2}$ using $12 \mathrm{~mm}$ flat punches.

\section{Post compression parameters of gastro-bilayer floating tablets}

\section{Weight variation}

Twenty tablets were randomly selected from each batch and calculated the percentage deviation of individual tablet weight from average weight of tablets.

\begin{tabular}{|c|c|c|c|c|c|c|c|}
\hline $\begin{array}{c}\text { Formulation } \\
\text { Code }\end{array}$ & $\begin{array}{l}\text { Drug } \\
\text { (mg) }\end{array}$ & $\begin{array}{c}\text { HPMC } \\
\text { K4 } \\
(\mathrm{mg})\end{array}$ & $\begin{array}{c}\text { HPMC } \\
\mathrm{K} 15(\mathrm{mg})\end{array}$ & $\begin{array}{c}\text { HPMC } \\
\text { K100 (mg) }\end{array}$ & $\begin{array}{l}\text { MCC } \\
\text { (mg) }\end{array}$ & $\begin{array}{c}\text { Magnesium } \\
\text { Stearate } \\
\text { (mg) }\end{array}$ & $\begin{array}{l}\text { Total } \\
(\mathrm{mg})\end{array}$ \\
\hline SR1 & 50 & 50 & - & - & 292 & 8 & 400 \\
\hline SR2 & 50 & 100 & - & - & 242 & 8 & 400 \\
\hline SR3 & 50 & 150 & - & - & 192 & 8 & 400 \\
\hline SR4 & 50 & - & 50 & - & 292 & 8 & 400 \\
\hline SR5 & 50 & - & 100 & - & 242 & 8 & 400 \\
\hline SR6 & 50 & - & 150 & - & 192 & 8 & 400 \\
\hline SR7 & 50 & - & - & 50 & 292 & 8 & 400 \\
\hline SR8 & 50 & - & - & 100 & 242 & 8 & 400 \\
\hline SR9 & 50 & - & - & 150 & 192 & 8 & 400 \\
\hline
\end{tabular}




\begin{tabular}{|c|c|c|c|c|}
\hline Ingredients (mg) & GBF & GBF0 & GBF1 & GBF2 \\
\hline $\begin{array}{l}\text { Immediate release layer } \\
\text { SD equivalent to } \\
10 \text { mg of ezetimibe (ESD1) }\end{array}$ & 40 & 40 & 40 & 40 \\
\hline $\operatorname{ccs}$ & 15 & 15 & 15 & 15 \\
\hline Lactose & 44 & 44 & 44 & 44 \\
\hline Magnesium stearate & 1 & 1 & 1 & 1 \\
\hline $\begin{array}{c}\text { Sustained release layer } \\
\text { Atenolol }\end{array}$ & 50 & 50 & 50 & 50 \\
\hline HPMC K100 & 150 & 150 & 150 & 150 \\
\hline Sodium bicarbonate & 15 & 20 & 25 & 30 \\
\hline $\mathrm{MCC}$ & 177 & 172 & 167 & 162 \\
\hline Magnesium stearate & 8 & 8 & 8 & 8 \\
\hline Total & 500 & 500 & 500 & 500 \\
\hline
\end{tabular}

Each batch contains 50 tablets

\section{Crushing strength}

Crushing strength was determined using Monsanto type hardness tester by selecting randomly six tablets from each batch.

\section{Friability}

Friability was conducted by considering tablets whose weight equivalent to $6.5 \mathrm{~g}$ using Roche friabilator apparatus (Model: 40 FT A01). It was rotated for $4 \mathrm{~min}$ at $25 \mathrm{rpm}$ where the tablets were allowed to fall from 6 inches height in each turn within the apparatus. These tablets were weighed to calculate percentage friability.

\section{Drug content}

Twenty tablets were randomly selected and crushed for the estimation of drug content. Powder weight equivalent to $50 \mathrm{mg}$ was transferred into $50 \mathrm{ml}$ volumetric flask and made to the volume by $0.1 \mathrm{M} \mathrm{HCl}$. The flask was placed in a sonicator till drug completely soluble. The solution was filtered through a filter paper $(0.45 \mu \mathrm{m}$ pore size) from this $1 \mathrm{ml}$ was taken and transferred to $25 \mathrm{ml}$ volumetric flask which was made up to the mark by $0.1 \mathrm{M} \mathrm{HCl}$. The absorbance of the solution was measured using UV-Visible spectrophotometer (Agilent, Cary 60) against the blank $0.1 \mathrm{M} \mathrm{HCl}$ at $232 \mathrm{~nm}$.

\section{In vitro floating ability}

The buoyancy capability of floating tablets was visually determined by employing the method described by Rosa et al. ${ }^{21}$ Buoyancy test was carried out by placing tablet in beaker containing $250 \mathrm{ml}$ of $0.1 \mathrm{M} \mathrm{HCl}$. The time required for floating the tablets to appear on to the surface of a dissolution medium (floating lag time, FLT) and the time during which the dosage form constantly float (total float time, TFT) were measured.

\section{Dissolution studies of gastro-bilayer floating tablets}

Dissolution study was carried out in triplicate for the developed gastro-bilayer floating tabletsusing USP II dissolution test apparatus (Electrolab, Mumbai, Model no. TDT-08L). In dissolution experiments, paddle rotational speed of $50 \mathrm{rpm}$ with $900 \mathrm{ml}$ of $0.1 \mathrm{M} \mathrm{HCl}$ dissolution medium was maintained at $37 \pm 0.5^{\circ} \mathrm{C}$ throughout the study. At predetermined time intervals $5 \mathrm{ml}$ of samples were withdrawn for a period of $12 \mathrm{~h}$ and filtered through a membrane filter $(0.45 \mu \mathrm{m}$, Millipore) . The volume was replaced with equal volume of fresh dissolution medium maintained at $37 \pm 0.5^{\circ} \mathrm{C}$ after each sampling. The samples withdrawn were analyzed by using a UV-Visible spectrophotometer at $232.4 \mathrm{~nm}$.

\section{Release kinetics ${ }^{22-24}$}

In vitro drug release data was subjected to mathematical models like zero order, first order, Higuchi, Hixon Crowell, Korsmeyer and Peppas in order to investigate the release pattern of optimized batch.

\section{RESULTS AND DISCUSSION}

Ezetimibe is practically insoluble in water (BCS class II). It has insufficient dissolution rate which is the limiting factor in the oral bioavailability. It has slow absorption leading to inadequate, variable bioavailability and gastrointestinal mucosal toxicity. So, to meet the aim of the study an attempt was made to improve the solubility of ezetimibe by SD technique. Nisha (2016) et al.25 improved the solubility of ezetimibe by preparing SD using PVP K-30 and PEG 6000 by SM. Therefore, for improving the solubility of ezetimibe, SDs techniques were adopted.

\section{FTIR}

The FTIR spectra of ezetimibe, PVP K-30 and SDs were displayed in Fig 1. Ezetimibe was characterized by -OH stretching at $3224.1 \mathrm{~cm}^{-1},-\mathrm{C}=\mathrm{O}$ stretching bands of lactam ring at $1712.7 \mathrm{~cm}^{-1}$ and $-\mathrm{C}=\mathrm{C}$ stretching band of aromatic ring at $1507.7 \mathrm{~cm}^{-1}$, -C-F stretching band at $1217.0 \mathrm{~cm}^{-1}$, -C-O stretching band at $1403.3 \mathrm{~cm}^{-1}$ (Figure 1A). Nearly similar results were reported by Yasser et al..$^{26}$ The FTIR spectrum of SD showed similar characteristic absorption peak of ezetimibe and PVP K-30. This indicates no new peaks or major shifting of the peaks denotes that the selected the drug and polymer/excipient are chemically compatible to each other. The physical interaction was necessary for the enhancement of solu- 


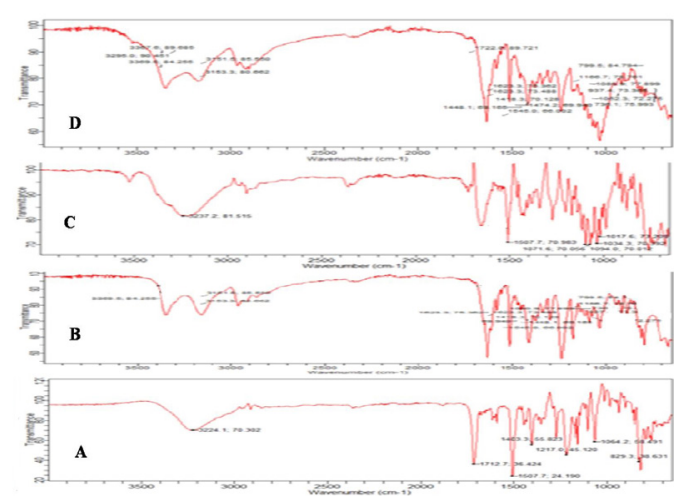

Figure 1: FTIR absorption spectra of A) ezetimibe, B) atenolol, C) ezetimibe SD (ESD1) and D) optimized bilayer tablet.

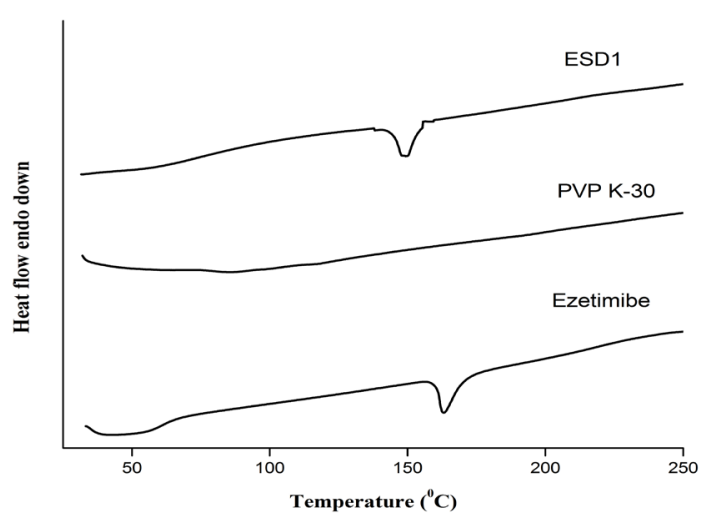

Figure 2: DSC thermograms of ezetimibe, PVP K-30 and ezetimibe SD (ESD1).

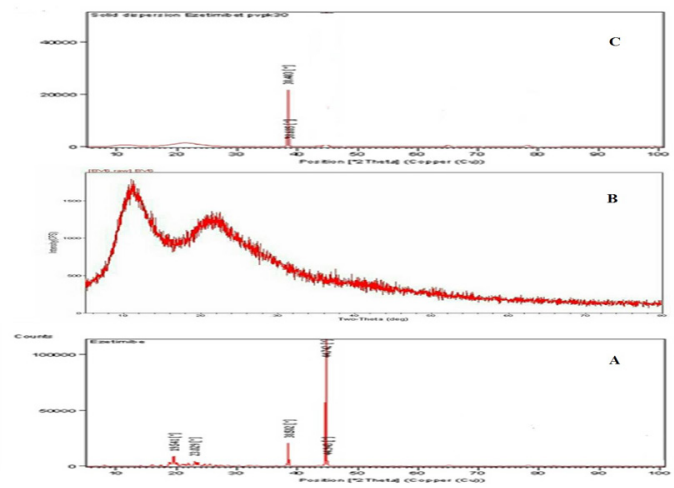

Figure 3: XRD pattern of A) ezetimibe, B) PVP K-30 and C) ezetimibe SD (ESD1).

bility of ezetimibe drug. The intensities of these peaks were reduced in SD mixture and slight shift in the peak values $\left(1709.27 \mathrm{~cm}^{-1}\right)$. The physical interaction was identified with PVP K-30 which might be helpful for the enhancement of the solubility of ezetimibe. The FTIR of the bilayer tablet showed no changes in the characteristics of the drugs. This indicates the compatibility between the drugs and excipients.

\section{DSC}

The DSC thermogram of pure drug ezetimibe showed endothermic peak at $162.66{ }^{\circ} \mathrm{C}$ (Figure 2) corresponds to its melting point. This indicates the drug was crystalline in nature. The broadness and shifting of the melting peaks of the drug ezetimibe in SD and decrease in heat of enthalpy indicates the drug was converted into the amorphous state (Figure 2). It confirms that ezetimibe was completely dispersed in the carrier. This was supported by the XRD study. Amorphous states have more surface area and interaction with the solvent. This might be helpful for the enhancement of solubility and dissolution rate of the ezetimibe.

\section{XRD}

The XRD diffractogram patterns of pure drug ezetimibe, PVP K-30 and SD were shown in Figure 3. XRD patterns of pure drug ezetimibe showed sharp and strong intense peaks at $2 \theta$ equivalent to $19.54^{\circ}, 23.02^{\circ}$, $38.50^{\circ}, 44.34^{\circ}$ and $44.74^{\circ}$ (Figure $3 \mathrm{~A}$ ). This indicates the strong crystalline nature of ezetimibe. PVP K-30 did not show any characteristics intense peaks at $2 \theta$, indicates the selected polymer was amorphous in nature (Figure 3B). The intensity of peaks at $2 \theta$ equivalent to $38.50^{\circ}$ was reduced indicating the conversion to amorphous state. The disappearance of the peaks at $2 \theta$ equivalent to $19.54^{\circ}, 23.02^{\circ}, 44.34^{\circ}$ and $44.74^{\circ}$ (Figure 3C) suggested the transformation to amorphous state. The peaks at $2 \theta$ equivalent to $19.54^{\circ}, 23.02^{\circ}$ becomes broad. This indicates crystalline nature of ezetimibe was converted to amorphous form. This was supported by DSC study and dissolution study. Amorphous product might be helpful to enhance the solubility and dissolution rate of ezetimibe.

\section{In vitro dissolution studies}

The dissolution profiles of pure drug ezetimibe and SDs were shown in Figure 4. The dissolution value of ezetimibe within $60 \mathrm{~min}$ showed an incomplete drug release $(21.65 \pm 0.48 \%)$. The dissolution profile of SDs showed higher dissolution rate than that of pure drug. Within 5 min of dissolution SD (ESD3) showed approximately 3.39 fold enhanced drug release. There was $59.11 \%$ drug release within 30 min of dissolution. This was approximately 4.04 fold enhancement of drug release than pure drug. It showed $81.64 \%$ drug release within $60 \mathrm{~min}$ of dissolution. The similarity factor $\left(\mathrm{f}_{2}\right)$ was found to be 16.38. This indicated the release profile of the SD was different from the pure drug. This was significant $(p<0.05)$. The enhancement of dissolution of ezetimibe in SDs may be due to the 


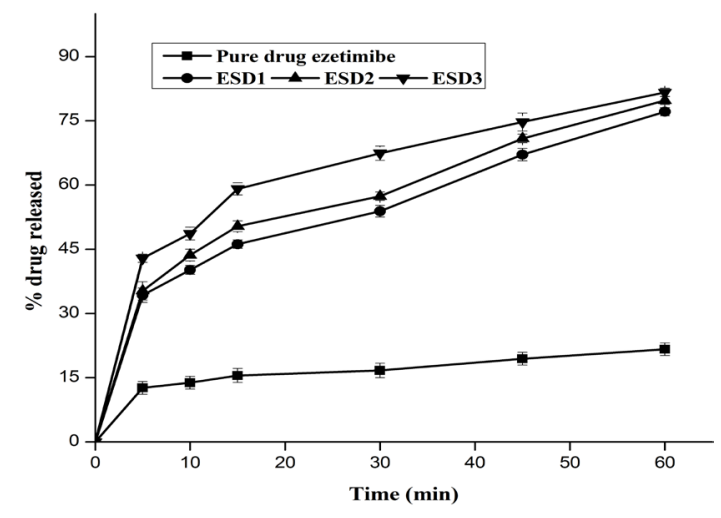

Figure 4: In vitro release profiles for solubility enhancement of ezetimibe.

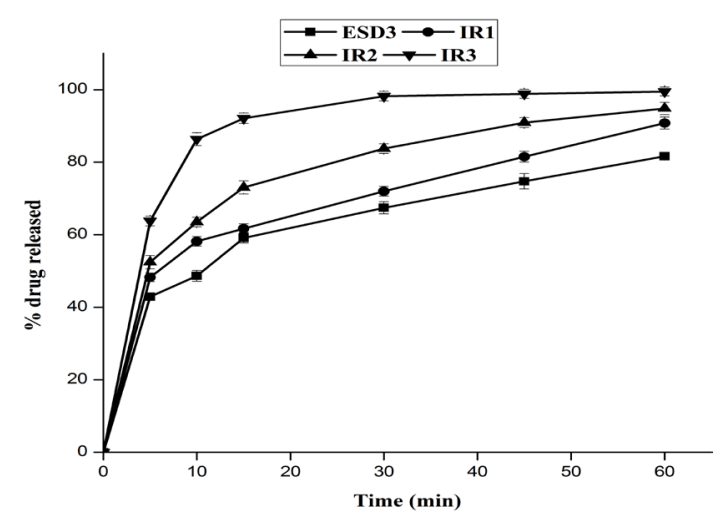

Figure 5: In vitro release profiles of immediate release tablets.

amorphous nature of the drug. Higher free energy of amorphous ezetimibe might have helped better interaction with solvent, leading to enhancement in dissolution. It was supported by XRD and DSC. SD equivalent to $10 \mathrm{mg}$ of ezetimibe (ESD3) was taken and formulated into immediate release tablets by varying the concentrations of superdisintegrants, croscarmellose sodium $(5,10$ and $15 \% \mathrm{w} / \mathrm{w})$. The powder blends were compressed in direct compression method.

Dissolution profiles of the prepared formulations were depicted in Figure 5. The formulations IR1 and IR2 showed $90.78 \%$ and $94.82 \%$ drug release within $60 \mathrm{~min}$. IR3 formulation containing $15 \% \mathrm{w} / \mathrm{w}$ of croscarmellose sodium showed $99.49 \%$ within $60 \mathrm{~min}$. It showed $92.08 \%$ of drug release within 15 min (Figure 5). This show nearly 5 fold more dissolution rate when compared to a pure drug over a period of $60 \mathrm{~min}$. This may be due to formulation encounters the dissolution medium, croscarmellose sodium in the immediate release layer of bilayer tablet swells by absorbing the liquid medium by wicking ${ }^{27}$ thereby liberating ezetimibe with fine dispersion. Therefore, the IR3 formulation containing $15 \% \mathrm{w} / \mathrm{w}$ of croscarmellose sodium was selected as immediate release layer in the preparation of gastro- bilayer floating tablets.

Atenolol is a class III drug with high solubility and low permeability with a half-lie of 6-7 h. Therefore, polymer should be incorporated into the formulation to retard the release. HPMC is a hydrophilic polymer and is strong enough to retard the release depending upon its viscosity and concentration. ${ }^{28}$ Formulations with three different grades of HPMC (K4, K15 and $\mathrm{K} 100)$ in three different concentrations $(12.5,25$ and $37.5 \% \mathrm{w} / \mathrm{w}$ ) along with other excipients were accurately weighed. The powder blends were subjected for sustained released tablet in direct compression method. The release profiles of the sustained released formulation (SR1-SR9) were illustrated in Figure 6. Hydrophilic nature of HPMC facilitates penetration of dissolution medium into the network structure of polymer chain thereby causing hydration of the polymer and swelling ${ }^{18}$ formed gelatinous layer act as boundary for the drug that must be released. The formulations with HPMC K 100 (SR7, SR8, SR9) showed $100 \%$ of drug release within 9-12 h whereas HPMC K 4 (SR1, SR2, SR3) and HPMC K15 (SR4, SR5, SR6) showed $100 \%$ drug release within 5-7 h respectively which showed in Figure 6. This showed that HPMC K4 and HMC K15 polymers unable to maintain their integrity for longer period when compared to HPMC K100. Therefore, HPMC K100 showed better retardant effect when compared to other two polymers (HPMC K4 and HPMC K15) over a period of $12 \mathrm{~h}$. Among three different concentrations of HPMC K100 the formulation with $37.5 \% \mathrm{w} / \mathrm{w}$ of polymer (SR9) showed good release profile over a period of $12 \mathrm{~h}$. This may be due to increased concentration of polymer may increase the thickness of gel barrier and tortuosity. It follows release profile as per Robinson and Eriksen equation i.e.,30-35 \% of drug release within $1 \mathrm{~h}$ and $60-65 \%$ released within $6 \mathrm{~h}$ and remaining drug is released after $12 \mathrm{~h} .{ }^{29}$ Therefore, SR9

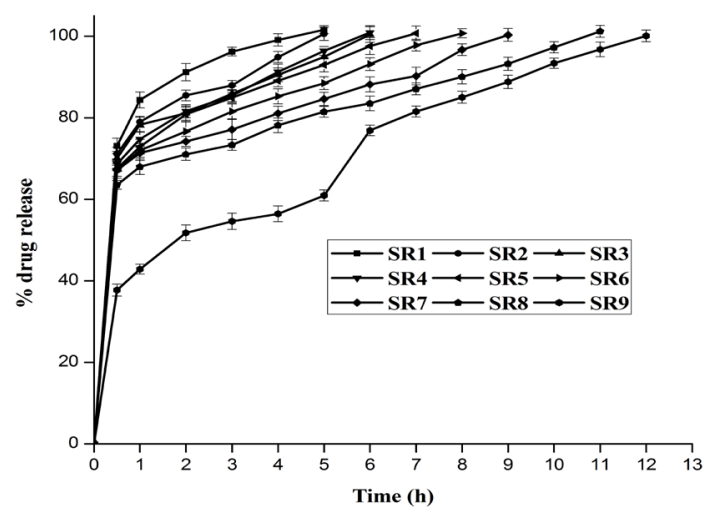

Figure 6: In vitro release profiles of sustained release tablets. 
formulation was selected as the optimized formulation. So, SR9 formulation was selected as sustained release layer in the preparation of gastro-bilayer floating tablets. Gastro- bilayer floating tablets were prepared by considering the formulations that showed optimized release profiles from the immediate release layer (IR3) and sustained release layer (SR9) with varying concentrations of sodium bicarbonate by direct compression method and shown in Table 3. The prepared formulations were evaluated for post compression parameters.

\section{POST-COMPRESSION PARAMETERS}

General appearance of prepared bilayer tablets was elegant. Differentiation between two layers made by giving blue colour to the immediate release layer and white colour to the sustained layer as shown in Figure 7.

\section{Weight variation}

Weight variation test is performed to check whether uniform weight is maintained among all the formulation batches. It is affected by the flow properties of the power blend. As weight of the prepared tablet was $500 \mathrm{mg}$, percentage deviation allowed was $5 \%$ as per Indian Pharmacopeia (IP). The prepared tablets were found to be in the range of $496-500 \mathrm{mg}$ (Table 4). So, these results suggested that weight variation was within the range and there was no significant variation in weight between different batches of tablets. This showed uniform die filling during tablet compression.

\section{Hardness}

Hardness gives an idea regarding how far the tablets resist to capping, abrasion or breakage under conditions of storage, transportation and handling. It affects drug dissolution and release to some extent. The hardness of all the formulations were within the range of 5.5-5.6 kg/ $\mathrm{cm}^{2}$ (Table 4). Therefore, all formulations were within the range. All the batches were found to have good thickness, dissolution and had the ability to withstand the handling abrasion.

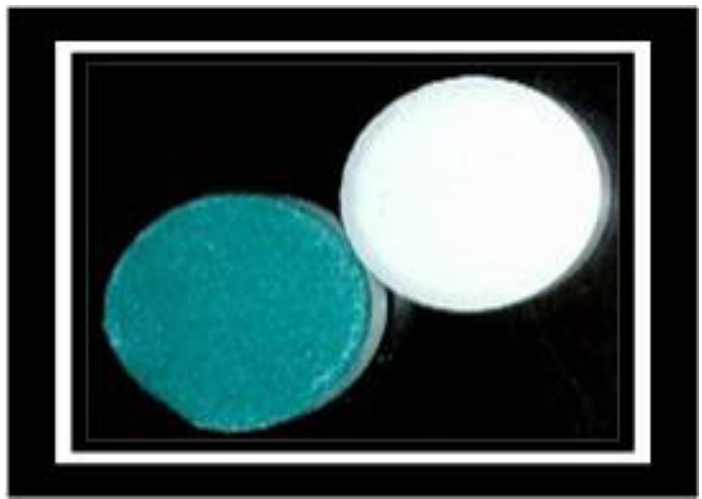

Figure 7: General appearance of prepared gastro- bilayer floating tablets. Immediate release layer (blue) and sustained release layer (white).

\section{Friability}

Friability test gives information regarding percentage loss in weight by tablets due to mechanical stress. Therefore, friability test decides whether the prepared tablets were withstanding the mechanical stress and confirms their suitability for processing. Friability of the prepared tablets were found to be in the range of $0.459-0.612$ (Table 4). As friability was below $1 \%$ indicating prepared tablets in each formulation can withstand the mechanical shocks.

\section{Drug content}

Drug content estimation helps in ensuring the consistency of dosage units, in which each unit in a batch should have drug content as per the limits of IP. Assay of gastro-bilayer floating tablets was with the help of UV-Visible Spectrophotometer and the content was estimated. Drug content for all the prepared formulation batches were found in the range of $99.18-100.12 \%$ for ezetimibe and 100.13-100.2\% for atenolol (Table 4) and the values obtained as per IP.

\section{In vitro floating ability}

Ideally, least possible FLT and continuous flotation of the dosage form in the upper GIT by preventing the dosage form from escaping to the lower GIT is necessary

\begin{tabular}{|c|c|c|c|c|c|c|c|}
\hline \multirow{2}{*}{$\begin{array}{l}\text { Formulation } \\
\text { code }\end{array}$} & \multirow{2}{*}{$\begin{array}{c}\text { Weight } \\
\text { variation }{ }^{\mathrm{a}}(\mathrm{mg})\end{array}$} & \multirow{2}{*}{$\begin{array}{c}\text { Hardness }^{b} \\
\left(\mathrm{~kg} / \mathrm{cm}^{2}\right)\end{array}$} & \multirow[t]{2}{*}{ Friability $^{c}$} & \multicolumn{2}{|c|}{ Drug content $(\%)^{d}$} & \multirow{2}{*}{$\begin{array}{l}\text { FLT } \\
(\min )\end{array}$} & \multirow{2}{*}{$\begin{array}{l}\text { TFT } \\
\text { (h) }\end{array}$} \\
\hline & & & & Ezetimibe & Atenolol & & \\
\hline GBF & $497 \pm 1.13 \%$ & $5.6 \pm 0.21$ & 0.517 & $99.18 \pm 1.18$ & $100.13 \pm 1.23$ & 45 & 7 \\
\hline GBF0 & $499 \pm 1.74 \%$ & $5.6 \pm 0.19$ & 0.504 & $99.95 \pm 1.71$ & $99.85 \pm 1.72$ & 32 & 7 \\
\hline GBF1 & $496 \pm 1.10 \%$ & $5.6 \pm 0.17$ & 0.612 & $99.85 \pm 1.11$ & $99.56 \pm 0.93$ & 15 & 8 \\
\hline GBF2 & $500 \pm 1.11 \%$ & $5.5 \pm 0.19$ & 0.459 & $100.12 \pm 0.99$ & $100.21 \pm 0.95$ & 2 & 12 \\
\hline
\end{tabular}

$\mathrm{a}: \mathrm{Avg} \pm \%$ deviation, $n=20$; b: mean $\pm \mathrm{SD}, n=6 ; \mathrm{c}: n=6.5 \mathrm{~g}$; : mean $\pm \mathrm{SD}, n=20$ 
for providing local drug absorption in the stomach. Therefore, the concentration of sodium bicarbonate was critical factor to arrive at the shortest lag time and to prolong the gastric retention time. This may be due to sodium bicarbonate can decrease the density of the tablets as it evolved $\mathrm{CO}_{2}$ bubbles on reaction with $\mathrm{HCl}$, resulting in the formation of pores due to entrapment of bubbles in the swollen polymer matrices of HPMC which ultimately helped the dosage form to float up to the surface of the medium. In addition, by using HPMC persistent buoyancy was achieved. In vitro floating ability was determined by considering the sodium bicarbonate in different concentrations $6.25 \% \mathrm{w} / \mathrm{w}, 7.5 \%$ w/w. Stages of floating of optimized formulation was shown in Figure 8. The tablet swelled by absorbing the solution at $30 \mathrm{sec}$. At $60 \mathrm{sec}$ it further absorbing the solution, swelled and tilted. It was partially floated with partial separation of immediate release layer $(80 \mathrm{sec})$. At $121 \mathrm{sec}$ tablet floated and the immediate release layer was completely separated from the tablet. The formulations (GBF and GBF0) showed FLT of $45 \mathrm{~min}$ and $32 \mathrm{~min}$ and floated up to $7 \mathrm{~h}$. The FLT $(15 \mathrm{~min}$ ) was decreased and floating time was increased as the concentration of the sodium carbonate was increased $(6.25 \% \mathrm{w} / \mathrm{w})$. Accordingly an increased concentration decreased FLT from $30 \mathrm{~min}$ to $2 \mathrm{~min}$. The formulation (GBF2) was considered as the optimized formulation as it has good FLT ( 2 min) along with TFT (up to $12 \mathrm{~h}$ ) (Table 4).

\section{In vitro dissolution studies}

Dissolution profile of the immediate release layer in bilayer floating tablet showed more than $92 \%$ ezetimibe release within $15 \mathrm{~min}$ as shown in Figure 9. Atenolol has absorption window in upper GIT whereas poor absorption in lower GIT. This varied absorption results in lowering bioavailability i.e., $50 \%$ with a half-life of 6-7 h. Therefore, was selected to formulate into floating layer. The dissolution profiles were shown in Figure 9. Increasing concentrations of sodium bicarbonate may produce higher level of effervescence that may result in increased rate of pore generation which may results in rapid hydration of the matrices and consequently faster drug release may occur. The formulation GBF, GBF0 and GBF1 showed $72 \%, 75 \%$ and $80 \%$ of drug release over a period of $12 \mathrm{~h}$ respectively and shown in Figure 9. This may be due to increased rate of pore generation which results in increased rate of drug release. The formulation containing $7.5 \% \mathrm{w} / \mathrm{w}$ of sodium bicarbonate (GBF2) showed complete drug release over a period of $12 \mathrm{~h}$ without losing the integrity of the tablet.

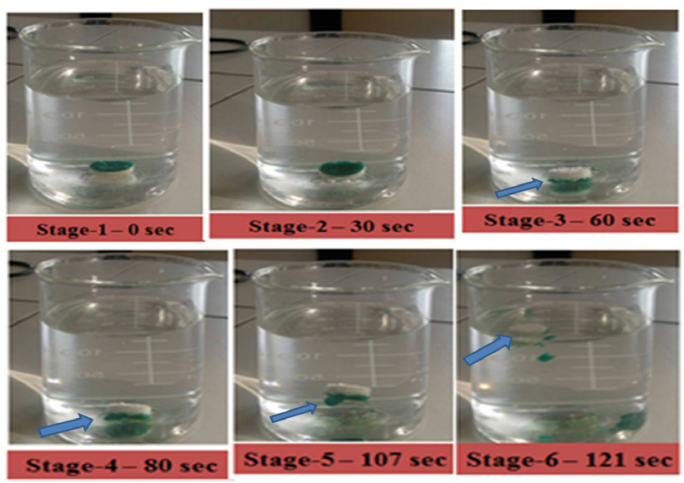

Figure 8: Stages of floating of the prepared gastro-bilayer floating tablets.

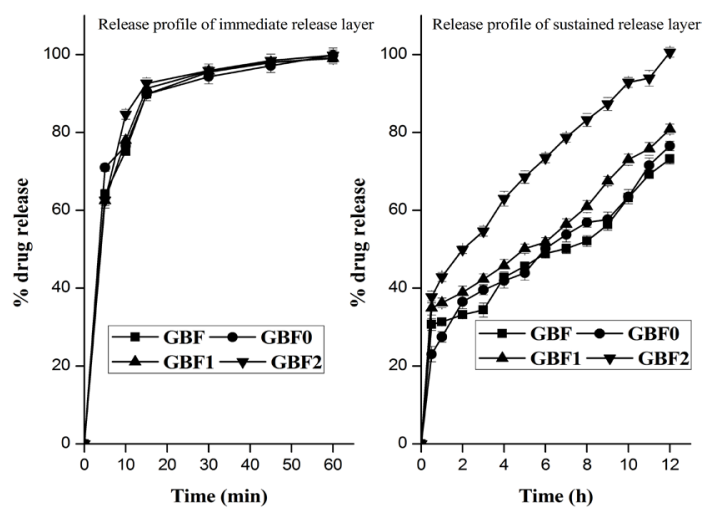

Figure 9: In vitro dissolution profile of gastro-bilayer floating tablets.

Therefore, GBF2 was considered as the optimized formulation.

\section{Kinetics of drug release}

Drug release kinetics were studied by using zero order, first order, Higuchi, Hixon Crowell, Koresmeyer and Peppas model to optimized formulation and shown in Table 5. Optimized formulation showed bi-phasic release pattern i.e., burst effect followed by sustained release. Burst effect may be due to highly hydrophilic nature of drug, polymer and method of preparation i.e., direct compression method that allows the drug to be on the surface of the tablet without entering the matrix. Therefore, when the tablet comes in contact with the dissolution medium, the drug present on the surface of the tablet entered into medium. Along with this free drug after $1 \mathrm{~h}$ chain relaxation also occurs for the polymers present at the surface releasing some amount of drug into the dissolution medium (42.86\%). So, burst effect is additive effect of the free drug present at the surface and the initial release of drug from the instantly swelled gel barrier. The result further can be supported by the fact that presence of sodium 
Table 5: Release kinetics of optimized formulation.

\begin{tabular}{|c|c|c|c|c|c|c|c|c|c|c|}
\hline \multirow{2}{*}{ Formulation } & \multicolumn{2}{|c|}{ Zero order } & \multicolumn{2}{|c|}{ First order } & \multicolumn{2}{c|}{ Higuchi } & \multicolumn{2}{c|}{$\begin{array}{c}\text { Korsmeyer and } \\
\text { Peppas }\end{array}$} & \multicolumn{2}{c|}{ Hixon Crowell } \\
\cline { 2 - 12 } & $\mathbf{r}^{2}$ & $\mathbf{K}_{0}$ & $\mathbf{r}^{2}$ & $\mathbf{K}_{1}$ & $\mathbf{r}^{2}$ & $\mathbf{K}_{\mathbf{H}}$ & $\mathbf{r}^{2}$ & $\mathbf{N}$ & $\mathbf{r}^{2}$ & $\mathbf{K}$ \\
\hline GBF2 & 0.870 & 6.340 & 0.859 & 0.077 & 0.974 & 25.61 & 0.969 & 0.355 & 0.761 & -0.011 \\
\hline
\end{tabular}

bicarbonate also leads to formation of bubbles facilitating the drug release during initial stages.

When the data was subjected to zero order and first order model higher $r^{2}$ value was obtained for zero order compared (0.870) to first order (0.859) is high, suggested that formulation follows zero order. This may be due to fact that depleted zone acts as reservoir matrix. The optimized formulation was studied for Higuchi and Hixon Crowell models where Higuchi model $r^{2}$ value (0.974) was found to be higher than Hixon Crowell value (0.761), which reveals that the release may follow diffusion mechanism. This may be due to hydrophilic nature of drug incorporated in a semi-solid matrix or sodium bicarbonate that forms gas bubbles on reaction with dissolution medium which creates porous system that allows the drug diffuses through the tortuous pathway created by porous system. Korsmeyer- Peppas model can be applied to any drug delivery system whose release mechanism was not well known or when more than one type of release is involved. Release exponent (n) value of optimized formulation was found to be 0.355 that indicates Fickian diffusion. HPMC being a swellable polymer when it comes in contact with dissolution medium it forms a gel (depletion layer model). Over a period of time, the release is being controlled by this gel layer which acts as a barrier for the drug release. Drug should pass through this barrier gel layer before entering into the dissolution medium. As the process of dissolution continuous, thickness of this barrier gel layer increases so, continuously drug is released and becomes more controlled.

\section{CONCLUSION}

Better hypertension and dyslipidaemia management needs concomitant drug treatment. Ezetimibe complete release was achieved through the $\mathrm{SD}$ technique using PVP K-30 (1:3\% w/w) and addition of superdisintegrants croscarmellose sodium. The total floating time of the optimized tablet was $12 \mathrm{~h}$ with 9 min of floating lag time. Hydroxypropyl methylcellulose K100 (37.5\% $\mathrm{w} / \mathrm{w})$ was optimized as release retardant polymer. Atenolol release was sustained through diffusion mechanism over $12 \mathrm{~h}$. It can be concluded that biphasic drug release pattern was successfully achieved through the formula- tion of gastro-floating bilayer tablets in this study, allowing strengthened combination therapy for hypertension and dyslipidemia.

\section{ACKNOWLEDGEMENT}

The authors would like to thank Aurobindo Pharma Pvt. Ltd., India. for supplying the pure drug ezetimibe. We are also thankful to MANSAS Educational Trust, Vizianagaram for providing required facilities to carry out this research work.

\section{CONFLICT OF INTEREST}

The authors declare no conflict of interest.

\section{ABBREVIATIONS}

APIs: Active pharmaceutical ingredients; BCS: Biopharmaceutical classification system; CCS: Croscarmellose sodium; DSC: Differential scanning calorimetry; FLT: Floating lag time; FTIR: Fourier transform infrared spectroscopy; GDDS: Gastroretentive drug delivery system; GIT: Gastro intestinal tract; HPMC: Hydroxyl propyl methyl cellulose; IR: Immediate release; MCC: Microcrystalline cellulose; NHANES: National health and nutritional examination survey; PEGs: Polyethylene glycols; PVP: Polyvinyl pyrrolidones; SDs: Solid dispersions; SM: Solvent evaporation method; SR: Sustained release; TFT: Total float time; XRD: X-ray diffraction analysis.

\section{REFERENCES}

1. Pawar VK, Kansal S, Garg G, Awasthi R, Singodia D, Kulkarni GT, et al. Gastroretentive dosage forms: A review with special emphasis on floating drug delivery systems. Drug Deliv. 2011;18(2):97-10.

2. Garg R, Gupta GD. Preparation and evaluation of gastroretentive floating tablets of silymarin. Chem Pharm Bull. 2009;57(6):545-9.

3. Guguloth M, Bomma R, Veerabrahma K. Development of sustained release floating drug delivery system for norfloxacin: in vitro and in vivo evaluation. PDA J Pharm SciTechnol. 2011;65(3):198-206.

4. He W, Li Y, Zhang R, Wu Z, Yin L. Gastro-floating bilayer tablets for the sustained release of metformin and immediate release of pioglitazone: Preparation and in vitro/in vivo evaluation. Int J Pharm. 2014;476(1-2):223-31.

5. Abebea A, Akselib I, Sprockela O, Kottalaa N, Cuitino AM. Review of bilayer tablet technology. Int J Pharm. 2014;461(1-2):549-58.

6. Fox CS. Cardiovascular disease risk factors, Type 2 diabetes mellitus and the Framingham heart study. Trends Cardiovasc Med. 2010;20(3):90-5.

7. http://www.who.int/whr/media_centre/factsheet3/en/ accessed on 23.07.2017. 
8. Dumbar RL. Hypertension, lipoprotein abnormalities and the metabolic syndrome. Advanced therapy in hyper tension and vascular disease. United States: B.C. Decker Inc. 2006;361-82.

9. Department of Health Statistics and Informatics. Causes of death 2008: data sources and methods. Geneva: World Health Organization. 2008.

10. Lim SS, Vos T, Flaxman AD, Danaei G, Shibuya K, Rohani HA, et al. A comparative risk assessment of burden of disease and injury attributable to 67 risk factors and risk factor clusters in 21 regions, 1990-2010: A systematic analysis for the global burden of disease study 2010. Lancet. 2012;380(9859):2224-60.

11. Dunbar RL. Hypertension, lipoprotein abnormalities and the metabolic syndrome. Advanced therapy in hypertension and vascular disease. $1^{\text {st }}$ ed. United States: B.C. Decker Inc. 2006;361-82.

12. Kai H, Kuwahara F, Tokuda K, Shibata R, Kusaba K, Niiyama H, et al. Coexistence of hypercholesterolemia and hypertension impairs adventitial vascularization. Hypertension. 2002;39(2):455-9.

13. Dey S, Mazumder B, Chattopadhyay S, Das MK, Sinha S, Ganguly S, et al. Polymers derived from Xanthomonascampesteris and Cyamopsistetragonolobus used as retardant materials for the formulation of sustained release floating matrix tablet of atenolol. Int J BiolMacromol. 2014;65:346-56

14. Mcanish J, Simpson WT, Homes BF, Young J, Elis SH. Bioavailability of atenolol formulatioins. Biopharm Drug Dispos. 1980;1(6):323-32.

15. Sahilesh T, Suesh K, Patel N. Formulation and evaluation of selfnanoemulsifying powder of ezetimibe. J Pharm Sci Drug Des. 2014;1(1):1-8.

16. Patel J, Sheehan V, Gurk C. Ezetimibe (Zetia): a new type of lipid-lowering agent. BUMC Proceedings. 2003;16(3):354-8.

17. Swain RP, Pendela S, Panda S. Formulation and evaluation of gastro-bilayer floating tablets of simvastatin as immediate release layer and atenolol as sustained release layer. Indian J Pharm Sci. 2016;78(4):458-68.
18. Kulkarni A, Bhatia M. Development and evaluation of regioselective bilayer floating tablets of atenolol and lovastatin for biphasic release profile. Iran J Pharm Res 2009;8:15-25.

19. Vo CL, Park C, Lee BJ. Current trends and future perspectives of solid dispersions containing poorly water-soluble drugs. Eur J Pharm Biopharm. 2013;85(3):799-813.

20. Vasconcelos T, Sarmento B, Costa P. Solid dispersions as strategy to improve oral bioavailability of poor water soluble drugs. Drug Disc Today. 2007;12(23-24):1068-75.

21. Rosa M, Zia H, Rhodes T. Dosing and testing in vitro of a bioadhesive and floating drug delivery system for oral application. Int J Pharm. 1994;105(1):65-70.

22. Higuchi T. Kinetic analysis of in vitro release rates of zidovudine matrix tablets. Theoretical analysis of rate release of solid drugs dispersed in solid matrices. J Pharm Sci. 1963;52:1145-9.

23. Korsemeyer RW, Gurny R, Doelker E, Buri P, Peppas NA. Kinetic analysis of in vitro release of zidovudine matrix tablets. Mechanism of solute release from porous hydrophilic polymers. Int J Pharm Sci. 1983;15(1):25-35.

24. Robinson JR, Eriksen SP. Theoretical formulation of sustained release dosage forms. J Pharm Sci. 1966;55(11):1254-63.

25. Nisha T, Kaoil K, Satish S. Development and characterization of solid dispersion of Ezetimibe by using solvent evaporation method. Asian J Pharm Pharmacology. 2016;2(2):44-9.

26. Ali Y, Shaimaa N, Alhammid A. Formulation and evauation of ezetimibe nanoparticles. Iraqi J Pharm Sci. 2015;24(2):11-21.

27. Palla S, Kotha RK, Paladugu A, Kumar RE, Adavi LS, Reddy RK. Bilayer floating tablets for gastro retentive drug delivery system. Int J Pharm Sci Nanotech. 2013;6(3):1097-2112.

28. Yin L, Qin C, Chen K, Zhu C, Cao H, Zhou J, et al. Gastro floating tablets of cephalexin: Preparation and in vitro/in vivo evaluation. Int $\mathrm{J}$ Pharm. 2013;452(1-2):241-8.

29. Robinson JR, Eriksen SP. Theoretical formulation of sustained release dosage forms. J Pharm Sci. 1966;55(11):1254-63.
PICTORIAL ABSTRACT

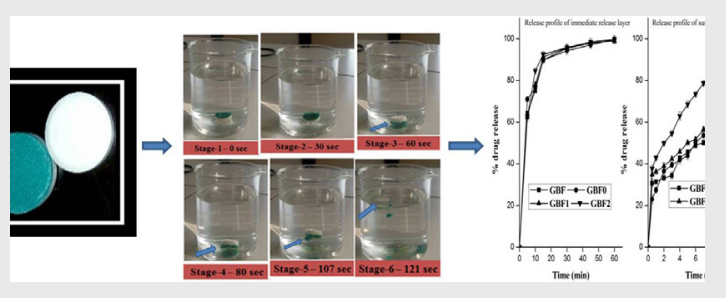

\section{ABOUT AUTHORS}

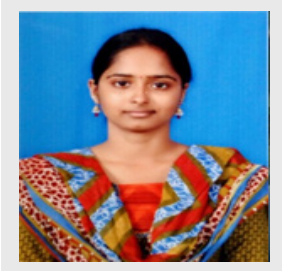

Avasarala Nagamani: She has a brilliant academic record with specialization in Pharmaceutical Technology from Maharajah's College of Pharmacy, Vizianagaram. She was a dedicated researcher and now working in Sri Krishna Pharmacuticals Ltd., Hyderabad.

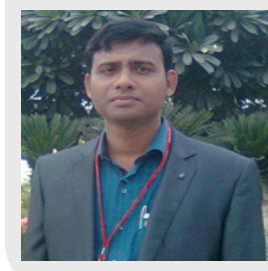

Dr. Ranjit Prasad Swain: Currently working as an Assistant Professor, Department of Pharmaceutical Technology at Maharajah's College of Pharmacy, Andhra Pradesh, India. His area of interest is bioavailability enhancement and novel drug delivery system. He has published 22 research papers in national and international journals. He presented 32 scientific papers in national and international conferences. He got best poster presentation award in $66^{\text {th }}$ Indian Pharmaceutical Congress (IPC) 2015. He is a Life member of Association of Pharmaceutical Teachers of India (A.P.T.I.) and member of IPA. 


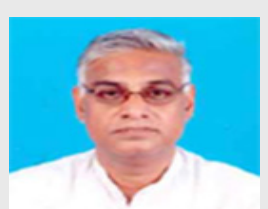

Prof. Panchagnula Udaya Shankar: Is presently working as Principal, Maharajah's College of Pharmacy, Andhra Pradesh, India. He has more than 30 years of industry and academic experience. He has been chaired many seminar and conferences. Prof. Uday Shankar was a visiting faculty at Kathamandu University, Nepal.

Cite this article: Swain RP, Nagamani A, Shankar PU. Formulation and Evaluation of Gastro-bilayer Floating Tablets of Ezetimibe as Immediate Release Layer and Atenolol as Sustained Release Layer. Indian J of Pharmaceutical Education and Research. 2019;53(2S):s93-s103. 